\title{
ANALYSIS OF BONDED ELASTIC BLOCKS
}

\author{
H.T. Banks \\ Center for Research in Scientific Computation \\ North Carolina State University \\ Raleigh, NC 27695-8205 USA
}

\author{
Gabriella A. Pinter \\ Department of Mathematical Sciences \\ University of Wisconsin Milwaukee \\ Milwaukee, WI 53201-0413
}

\author{
O.H. Yeoh \\ Lord Corporation \\ Mechanical Products Division \\ Erie, PA 16514-0038
}

\begin{abstract}
We investigate the compression of rubber blocks bonded to rigid metal plates for several different geometries, namely for rectangular and V-shaped blocks. We obtain reasonably simple solutions in series form relating the force and the displacement using the "pressure method" developed by Gent. Our initial assumption concerning the incompressibility of the rubber is subsequently relaxed to "near incompressibility" to yield more accurate solutions. We demonstrate a very good agreement between the theoretical solutions and the results of linear finite element simulations.
\end{abstract}

Key words: elasticity, compression, incompressibility, near incompressibility, partial differential equations, finite element simulations

\section{Introduction}

In this note we examine the compression of rubber blocks bonded to rigid metal plates for several specific geometries. Rigorous analysis of such components is nontrivial since the solutions are complicated. However, for design purposes it is most desirable to produce (if possible) reasonably simple closed form solutions for these geometries that provide a 
good approximation to the actual behavior of the components. A number of approximate methods have been developed using simplifying assumptions such as incompressibility of the rubber and linear elastic behavior. Here we adapt an approach developed by Gent, $[2,3,4]$, called the "pressure method". When a bonded block is compressed, the deformation can be considered to take place in two stages: a simple homogeneous compression, $\left(F_{1}\right)$, which is equivalent to compression between lubricated plates and subsequent shear deformations, $\left(F_{2}\right)$, that restore points in the planes of the bonded surfaces to their original positions. Hence, assuming superposition, the compression force is given by $F=F_{1}+F_{2}$. Solutions derived from the pressure method approach have been quite successful and are often quoted in rubber engineering design handbooks ([6]). As we described in [1], solutions using this method give very good approximations to the compressive force in the case of rectangular and annular blocks. As we shall demonstrate, the assumption of incompressibility can be relaxed and a good agreement can be established between the resulting theoretical formulation and the numerical finite element results for slightly compressible rectangular and annular blocks.

In this paper after a general introduction to the pressure method we revisit the case of a rectangular bonded block and present in detail the results for the slightly compressible case. Next we apply the pressure method to a V-shaped mount and extend the solutions to the slightly compressible case. At the end of this note we present a comparison of theoretical and finite element analysis predictions.

\section{The pressure method}

In this section we provide a brief introduction to the pressure method and present the general strategy used in obtaining the governing equations for different geometries. Other descriptions of the pressure method can be found in $[2,3,4]$. As stated in the introduction we use the assumption that the shear modulus is constant (i.e., there is a linear relationship between stress and strain) and thus our results apply for small strains. For small compressions the load-deflection relation may be written as:

$$
\frac{F}{A}=E f(S) \varepsilon
$$

where $A$ is the cross-sectional area, $f(S)$ is the shape function, $S=\frac{\text { bonded area }}{\text { unbonded area }}$, E is Young's modulus for the rubber and $\varepsilon$ is the average compressive strain. The goal from the designer's point of view is to compute $f(S)$ for different geometries. The deformation is considered to occur in two stages: first there is a simple homogeneous compression and then subsequent shear deformations restore points in the planes of the bonded surfaces to their original positions. We denote the compressive force resulting from the simple homogeneous compression by $F_{1}$. The compressive stress, $\sigma_{1}$, for the first deformation is uniform and given by:

$$
\frac{F_{1}}{A}=\sigma_{1}=E \varepsilon
$$


when displacements occur equally in the $x, y$ plane, or

$$
\frac{F_{1}}{A}=\sigma_{1}=\frac{E \varepsilon}{1-\nu^{2}},
$$

where $\nu$ is Poisson's ratio, when the displacements are restricted to one direction (e.g., long strip). For incompressible materials, $\nu$ is 0.5 so that $\frac{1}{\left(1-\nu^{2}\right)}=\frac{4}{3}$. Then for $\frac{F_{1}}{A}=E f_{1}(S) \varepsilon$, $f_{1}(S)$ ranges from 1 to $\frac{4}{3}$.

An empirical form for the $f_{1}(S)$ term (see [3]) is:

$$
f_{1}(S)=\frac{4}{3}-\frac{2}{3} \frac{a b+t^{2}}{a^{2}+b^{2}+2 t^{2}}
$$

where $2 a, 2 b$ are sides of the rectangular cross-section or axes of the elliptic cross-section. Note that this formula gives 1 when displacements occur equally in the $\mathrm{x}, \mathrm{y}$ plane (i.e., square block) and equals $\frac{4}{3}$ for an infinite block.
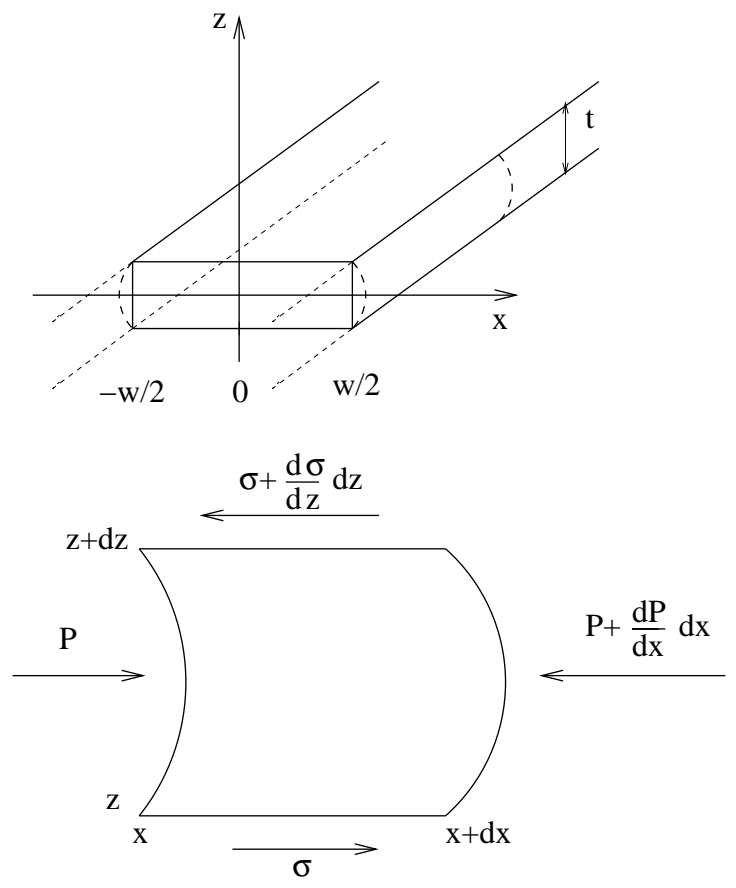

Figure 1: Forces on an element

According to Gent, under certain conditions, the stresses required to maintain the second deformation can be deduced readily by analogy with Reynolds' theory of thin lubricating films of a viscous liquid. For relatively thin blocks the stresses are represented in the form of a hydrostatic pressure $P$ that is maximal in the center of the block and falls to zero at the free surfaces. The governing relation for $P$ can be derived for different geometries. To illustrate the method we present the derivation of $P$ in detail for a thin, bonded infinite strip. 
Consider static equilibrium of forces on the element in Figure 1 where $P$ denotes the pressure and $\sigma$ denotes the shear stress in the $x, y$ plane:

$$
\frac{d P}{d x} d x d y d z=-\frac{d \sigma}{d z} d z d x d y
$$

and thus

$$
\frac{d P}{d x}=-\frac{d \sigma}{d z}
$$

Since the pressure is assumed constant through the thickness $\frac{d P}{d x}$ is independent of $\mathrm{z}$, which implies that

$$
\frac{d^{2} \sigma}{d z^{2}}=0
$$

The shear stress, $\sigma$, is given by

$$
\sigma=-G \frac{d k}{d z}
$$

where $k$ is the x-displacement of the volume element and $G$ is the shear modulus of the rubber $\left(\frac{E}{2(1+\nu)}\right)$.

From (2.4) it follows that

$$
\frac{d^{3} k}{d z^{3}}=0
$$

and

$$
\frac{d P}{d x}=G\left(\frac{d^{2} k}{d z^{2}}\right)
$$

Equation (2.6) indicates that an initially plane cross section takes a parabolic shape in the deformed state, with a profile given by:

$$
\frac{k}{k_{m}}=1-\left(\frac{2 z}{t}\right)^{2}
$$

where $k=0$ when $z= \pm \frac{t}{2}$ and $k=k_{m}$ when $z=0$. Substituting (2.8) into (2.7) we obtain:

$$
\frac{d P}{d x}=-8 G \frac{k_{m}}{t^{2}} .
$$

To find $k_{m}$ we equate the volume of the bulge with the volume displaced by the compression of the block lying within the plane at $x$ (see Figure 2). Only a unit length is considered in the case of an infinite block. 


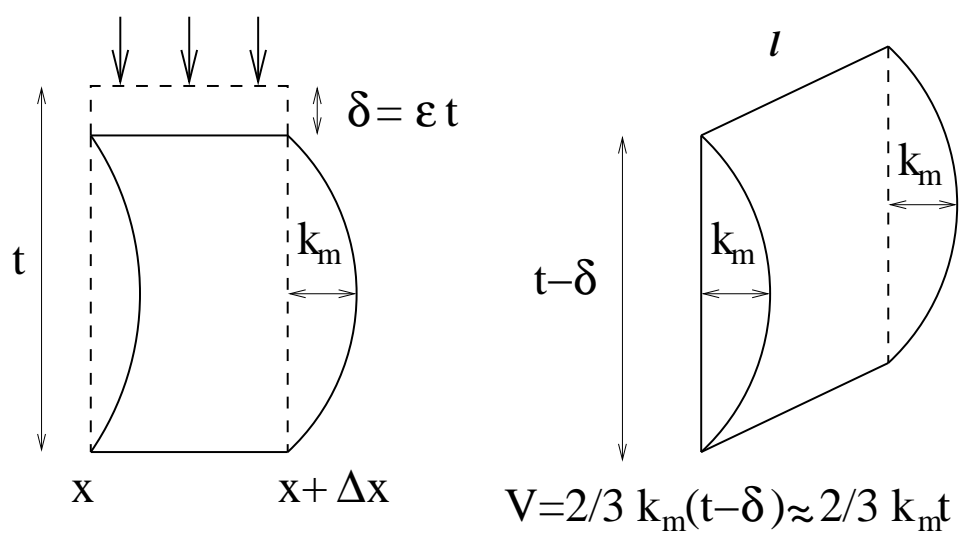

Figure 2: Volume of the bulge

The change in volume is given by

$$
\Delta V=\frac{2}{3} \Delta k_{m} t-\varepsilon t \Delta x .
$$

On the other hand,

$$
\frac{\Delta V}{V}=-\frac{P}{K},
$$

where $K$ is the bulk modulus of the material. Thus

$$
\frac{\Delta V}{V}=\frac{2}{3} \frac{\Delta k_{m} t}{\Delta x t}-\frac{\varepsilon t \Delta x}{\Delta x t}=-\frac{P}{K},
$$

which yields

$$
\begin{aligned}
\frac{2}{3} \frac{\Delta k_{m}}{\Delta x} & =\varepsilon-\frac{P}{K} \\
\frac{d k_{m}}{d x} & =\frac{3}{2}\left(\varepsilon-\frac{P}{K}\right) .
\end{aligned}
$$

Note that if $K=\infty$ (incompressible material) then $\frac{d k_{m}}{d x}=\frac{3}{2} \varepsilon$. Combining (2.9) and (2.10) we obtain

$$
\frac{d^{2} P}{d x^{2}}=-12 \frac{G}{t^{2}}\left(\varepsilon-\frac{P}{K}\right) .
$$

When deformation occurs in the $y$-direction as well we have

$$
\frac{\partial^{2} P}{\partial x^{2}}+\frac{\partial^{2} P}{\partial y^{2}}=-12 \frac{G}{t^{2}}\left(\varepsilon-\frac{P}{K}\right) .
$$


In objects with cylindrical symmetry we obtain

$$
\frac{d^{2} P}{d r^{2}}+\frac{1}{r} \frac{d P}{d r}=-12 \frac{G}{t^{2}}\left(\varepsilon-\frac{P}{K}\right) .
$$

If incompressibility is assumed then the equation is the same with the term $\frac{P}{K}$ removed (i.e., take $K=\infty)$. Once the pressure $P$ is found, the corresponding compressive force can be obtained by integration and $f_{2}(S)$ (the component of the shape function arising from the second step of the deformation) can be determined. Thus for a long bonded strip the solution of (2.11) with boundary conditions $P=0$ at $x= \pm w / 2$, and $d P / d x=0$ at $x=0$ yields

$$
P=\frac{6 G \varepsilon}{t^{2}}\left(\frac{w^{2}}{4}-x^{2}\right)
$$

in the incompressible case. Thus

$$
F_{2}=\int_{-w / 2}^{w / 2} P d x=\frac{G \varepsilon w^{3}}{t^{2}} .
$$

For the long bonded strip the force per unit length associated with the simple, homogeneous compression is given by

$$
F_{1}=\frac{4}{3} E \varepsilon w,
$$

where $E$ is the Young's modulus of the rubber. Thus,

$$
F=F_{1}+F_{2}=\frac{4}{3} E \varepsilon w\left(1+\frac{w^{2}}{4 t^{2}}\right)
$$

and since the shape factor for the long bonded strip is $S=\frac{w}{2 t}$, we obtain that the shape function is

$$
f(S)=\frac{4}{3}\left(1+S^{2}\right)
$$

As we noted in [1] Equation (2.12) is analogous to the equation governing the torsion of a prism. Solutions of the torsion problem exist for many different geometries $[7,8,9]$ and can be used directly.

Historically, the general incompressible problem was solved by Gent and Meinecke in 1970 [3]. In 1994 Gent extended his solutions for the infinite strip (1-D) and the disk (axis-symmetric geometry) to a compressible material [4]. The axis-symmetric solution was generalized by Kelly to the annulus [5]. Our work extends the incompressible solution for rectangular blocks to compressible material and uses this result to find solutions for $\mathrm{V}$ shaped rubber blocks. We also compare the theoretical results with numerical simulations using finite element methods. 


\section{Rectangular blocks}

In this section we review the compression of bonded rectangular blocks of length $l$, width $w$ and thickness $t$. In [1] we outlined the results for the incompressible and the slightly compressible case; here we present the analysis in detail for the latter case. Results for the former can be found in [1,3], but we note that they are not expected to provide a good approximation unless some compressibility correction is used especially when the shape factor, $S$, is large.

The compressive force $F_{1}$ corresponding to the pure homogeneous deformation is given by

$$
\frac{F_{1}}{A}=E \varepsilon f_{1}(S)
$$

where $f_{1}(S)$ is the empirical factor given by (2.1), with $a=\ell / 2$, and $b=w / 2$. The crosssectional area $A$ is $\ell w$. As noted in [1] for blocks of moderate to large shape factors $F_{1}$ is much smaller than $F_{2}$, so uncertainty in $f_{1}(S)$ is usually not critical.

We derive the force $F_{2}$ by analogy to Reynolds' squeeze film theory. In the slightly compressible case the pressure satisfies the equation

$$
\frac{\partial^{2} P}{\partial x^{2}}+\frac{\partial^{2} P}{\partial y^{2}}=-\frac{12 G}{t^{2}}\left(\varepsilon-\frac{P}{K}\right)
$$

with boundary conditions

$$
\begin{aligned}
& P=0 \text { at } x= \pm w / 2, \quad y= \pm \ell / 2, \quad \text { and } \\
& \frac{\partial P}{\partial x}=0, \quad \frac{\partial P}{\partial y}=0 \text { at } x=y=0,
\end{aligned}
$$

where $K$ is the bulk modulus of the rubber. Since the pressure is symmetric with respect to the $y$-axis and $P=0$ at $x= \pm w / 2$ we propose to find a solution to (3.18) in the form

$$
P(x, y)=\sum_{n=1,3,5, \ldots}^{\infty} a_{n} \cos \frac{n \pi x}{w} Y_{n}
$$

where $a_{n}, n=1,3,5, \ldots$ are constant coefficients and $Y_{1}, Y_{3}, \ldots$ are functions of $y$ only. Substituting (3.21) into (3.18), formally we obtain

$$
\begin{aligned}
& -\sum_{n=1,3,5, \ldots}^{\infty} a_{n} \frac{n^{2} \pi^{2}}{w^{2}} \cos \frac{n \pi x}{w} Y_{n}+\sum_{n=1,3,5, \ldots}^{\infty} a_{n} \cos \frac{n \pi x}{w} Y_{n}^{\prime \prime} \\
& =-\frac{12 G}{t^{2}}\left(\varepsilon-\frac{1}{K} \sum_{n=1,3,5, \ldots}^{\infty} a_{n} \cos \frac{n \pi x}{w} Y_{n}\right),
\end{aligned}
$$

which yields

$$
\sum_{n=1,3,5, \ldots}^{\infty}\left[a_{n} \cos \frac{n \pi x}{w} Y_{n}^{\prime \prime}-\left(a_{n} \frac{n^{2} \pi^{2}}{w^{2}} \cos \frac{n \pi x}{w}+\frac{12 G}{t^{2} K} a_{n} \cos \frac{n \pi x}{w}\right) Y_{n}\right]=-\frac{12 G \varepsilon}{t^{2}}
$$


We expand the right side in Fourier series for $-w / 2<x<w / 2$ to obtain

$$
-\frac{12 G \varepsilon}{t^{2}}=-\sum_{n=1,3,5, \ldots}^{\infty} \frac{12 G \varepsilon}{t^{2}} \frac{4}{n \pi}(-1)^{\frac{n-1}{2}} \cos \frac{n \pi x}{w}
$$

Thus $Y_{n}(y)$ satisfies the following ordinary differential equation

$$
Y_{n}^{\prime \prime}-\left(\frac{n^{2} \pi^{2}}{w^{2}}+\frac{12 G}{t^{2} K}\right) Y_{n}=-\frac{12 G \varepsilon}{t^{2}} \frac{4}{n \pi a_{n}}(-1)^{\frac{n-1}{2}}
$$

with

$$
\begin{aligned}
& Y_{n}( \pm \ell / 2)=0 \quad \text { and } \\
& Y_{n}^{\prime}(0)=0
\end{aligned}
$$

The general solution of $(3.24)$ is

$$
Y_{n}(y)=c_{1} \sinh \lambda_{n} y+c_{2} \cosh \lambda_{n} y+\frac{1}{\lambda_{n}^{2}} \frac{12 G \varepsilon}{t^{2}} \frac{4}{n \pi a_{n}}(-1)^{\frac{n-1}{2}}
$$

with

$$
\lambda_{n}^{2}=\frac{n^{2} \pi^{2}}{w^{2}}+\frac{12 G}{t^{2} K}
$$

Since the pressure is symmetric with respect to the $x$-axis, it follows that $c_{1}=0$. From (3.25) we obtain that

$$
c_{2}=\frac{1}{\lambda_{n}^{2}} \frac{12 G \varepsilon}{t^{2}} \frac{4}{n \pi a_{n}}(-1)^{\frac{n-1}{2}} \frac{1}{\cosh \frac{\lambda_{n} \ell}{2}} .
$$

We note that in this case (3.26) is also satisfied. Thus

$$
P=\frac{12 G \varepsilon}{t^{2}} \sum_{n=1,3,5, \ldots}^{\infty} \frac{4}{n \pi \lambda_{n}^{2}}(-1)^{\frac{n-1}{2}}\left[1-\frac{\cosh \left(\lambda_{n} y\right)}{\cosh \left(\lambda_{n} \ell / 2\right)}\right] \cos \frac{n \pi x}{w}
$$

with

$$
\lambda_{n}^{2}=\frac{n^{2} \pi^{2}}{w^{2}}+\frac{12 G}{t^{2} K}
$$

We integrate $P$ over the metal plate to obtain

$$
F_{2}=\int_{-\ell / 2}^{\ell / 2} \int_{-w / 2}^{w / 2} P d x d y=\frac{24 G \varepsilon}{t^{2}} \sum_{n=1,3,5, \ldots}^{\infty} \frac{4 w \ell}{n^{2} \pi^{2} \lambda_{n}^{2}}-\frac{8 w}{n^{2} \pi^{2} \lambda_{n}^{3}} \tanh \frac{\lambda_{n} \ell}{2} .
$$

This series converges rapidly and can be computed easily for different ratios of $w / l$.

Adding $F_{1}$ and $F_{2}$ yields

$$
\frac{F}{A}=\frac{F_{1}+F_{2}}{A}=\frac{1}{A}\left[E \varepsilon f_{1}(S)+\frac{24 G \varepsilon}{t^{2}} \sum_{n=1,3,5, \ldots}^{\infty} \frac{4 w \ell}{n^{2} \pi^{2} \lambda_{n}^{2}}-\frac{8 w}{n^{2} \pi^{2} \lambda_{n}^{3}} \tanh \frac{\lambda_{n} \ell}{2}\right] .
$$


This result was compared to numerical simulations using linear finite element methods in [1]. The half-width of the block $(w / 2)$ was kept constant at $0.0254 \mathrm{~m}(1.0 \mathrm{in})$ while the half length $(\ell / 2)$ and the thickness $t$ were varied. The material was assumed to be linearly elastic with Young's modulus of $4.137 \cdot 10^{6} \mathrm{~Pa},(600 \mathrm{psi})$ and a Poisson's ratio of 0.4995 (slightly compressible material). The theoretically and numerically predicted vertical force needed to compress the rectangular blocks are depicted in Figure 3. Discrepancy between the finite element results and theory is less than $10 \%$ which is very satisfying considering that both methods are approximate.

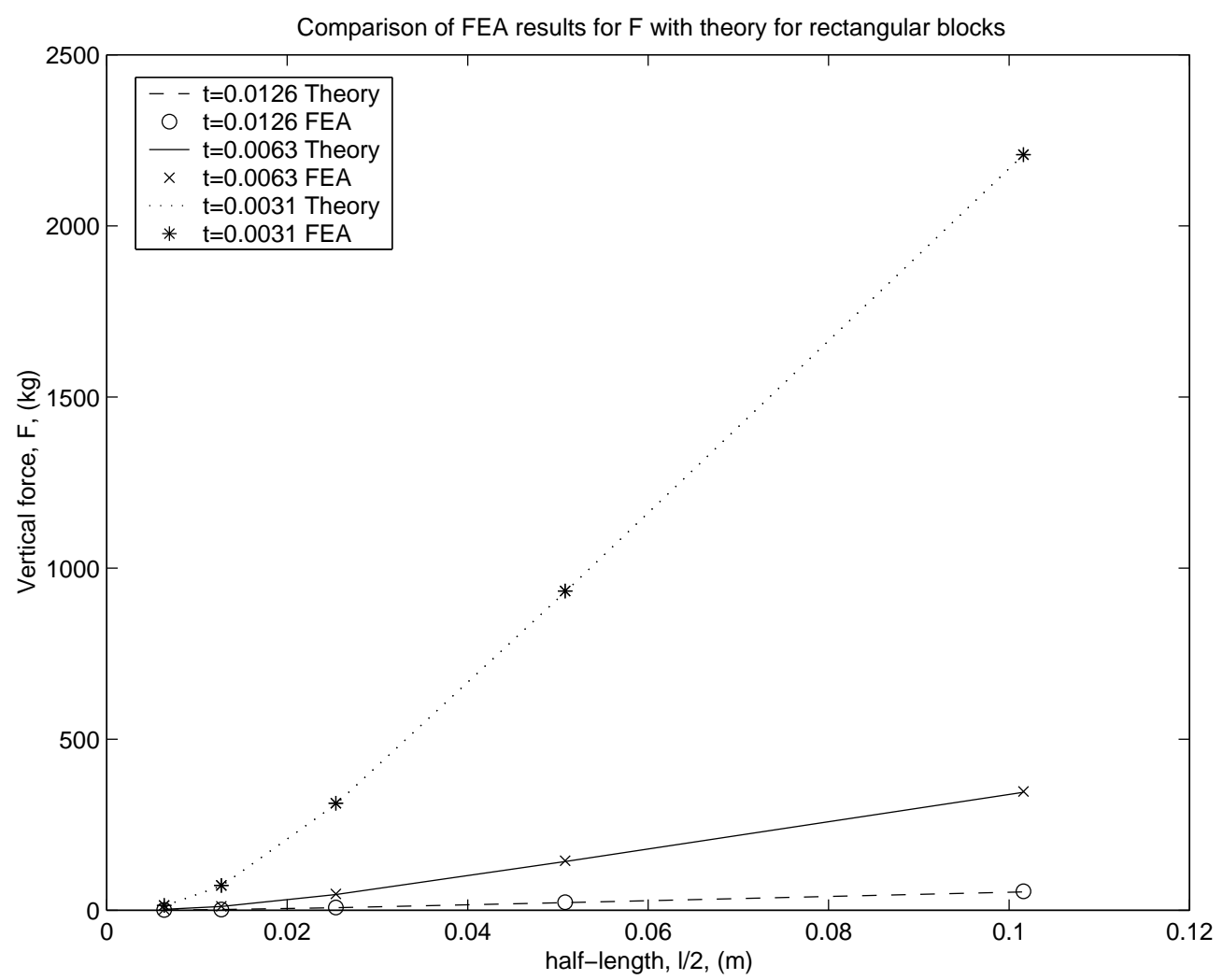

Figure 3: Comparison of FEA results for $F$ with theory for rectangular blocks

\section{V-shaped blocks}

In this section we derive the total axial force corresponding to a small vertical displacement $\delta$ for V-shaped blocks under different assumptions. V-shaped rubber blocks are used in rubber springs to achieve particular combinations of stiffnesses in the three orthogonal directions. They can be found for example in railway bogie suspensions. Our goal is to develop expressions for the shape function to aid in the design of such blocks by predicting the stiffness parameters. 


\subsection{Infinitely long V-shaped blocks}

We start by analyzing the case of infinitely long Chevron-shaped blocks. We assume that the length of the block is much larger than its width, thus we can neglect the displacements at the two ends. We also assume in this derivation that the rubber is incompressible. The cross-section of the V-shaped block is depicted in Figure 4. Since the block is symmetric, we consider only one leg of the Chevron-shaped mount first.

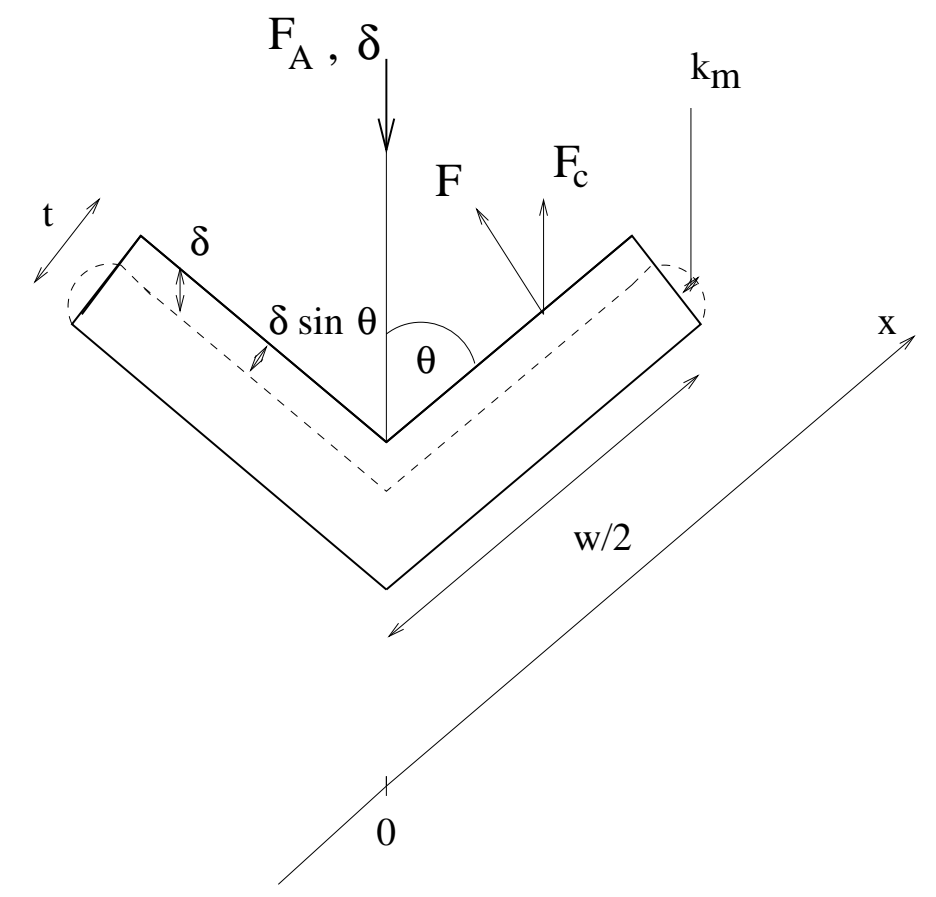

Figure 4: Cross-section of the infinitely long V-shaped block

The vertical displacement, $\delta$, may be resolved into its components $\delta \sin \theta$, which is normal to the metal plate and causes compression in the rubber, and $\delta \cos \theta$, which is tangential to the metal plate and causes shear. We want to calculate the vertical force per unit length, $F_{a}$, on one leg of the chevron. This is given by

$$
F_{a}=F_{c}+F_{s},
$$

where $F_{c}$ and $F_{s}$ are the contributions (per unit length) from the compression and shear deformations respectively. First we consider compression, which is assumed to take place in two stages as explained in the introduction. The force, $F_{1}$, associated with the simple, homogeneous compression is in the form given by

$$
\frac{F_{1}}{A}=\frac{4}{3} E \frac{\delta \sin \theta}{t}=\frac{4 G \delta \sin \theta}{t},
$$

with the cross-sectional area $A=w / 2$. Since incompressibility is assumed, we have $E=3 G$. 
To compute $F_{2}$ we again use the analogy to Reynolds' squeeze film theory. As we showed in Section 2 the local hydrostatic pressure $P$ sustaining the bulge profiles solves the ordinary differential equation

$$
\frac{\partial P}{\partial x}=-\frac{8 G k_{m}}{t^{2}}
$$

where $k_{m}$ is the maximal bulge (see Figure 2). The volume of the bulge is approximated by $V \approx \frac{2}{3} k_{m} t$, while the displaced volume per unit length is given by $V=x \delta \sin \theta$. Hence $k_{m}=\frac{3}{2 t} x \delta \sin \theta$.

Thus,

$$
\begin{aligned}
& \frac{\partial P}{\partial x}=-\frac{12 G x \delta \sin \theta}{t^{3}} \\
& P=0 \text { at } x= \pm \frac{w}{2}
\end{aligned}
$$

and the solution is

$$
P=\frac{6 G \delta \sin \theta}{t^{3}}\left(\frac{w^{2}}{4}-x^{2}\right) .
$$

The pressure acts on the metal plate and therefore the corresponding force per unit length, $F_{2}$, is obtained by integrating $P$ along the plate

$$
F_{2}=\int_{0}^{w / 2} \frac{6 G \delta \sin \theta}{t^{3}}\left(\frac{w^{2}}{4}-x^{2}\right) d x=\frac{G \delta \sin \theta w^{3}}{2 t^{3}}
$$

This implies that the compression force acting perpendicular to the metal plate is given by

$$
\begin{aligned}
F & =F_{1}+F_{2}=\frac{4}{3} E \frac{\delta \sin \theta w}{2 t}+\frac{G \delta \sin \theta w^{3}}{2 t^{3}} \\
& =\frac{4}{3} A E \frac{\delta \sin \theta}{t}\left(1+\frac{w^{2}}{4 t^{2}}\right)
\end{aligned}
$$

and the axial component $F_{c}$ of the compression force $F$ is

$$
F_{c}=F \sin \theta=\frac{4}{3} A E \frac{\delta \sin ^{2} \theta}{t}\left(1+\frac{w^{2}}{4 t^{2}}\right) .
$$

To find the total axial force $F_{a}$ we also need to compute the vertical component of the shear force induced by the tangential component of the displacement. The shear force can be determined as

$$
F_{t}=\frac{G A \delta \cos \theta}{t}
$$

and its contribution to the axial force is

$$
F_{s}=F_{t} \cos \theta=\frac{G A \delta \cos ^{2} \theta}{t}
$$


Thus the axial force on one leg of the V-shaped block is

$$
F_{a}=F_{s}+F_{c}=\frac{G A \delta \cos ^{2} \theta}{t}+\frac{4}{3} A E \frac{\delta \sin ^{2} \theta}{t}\left(1+\frac{w^{2}}{4 t^{2}}\right),
$$

which implies that the total axial force per unit length is

$$
F_{A}=2 F_{a}=\frac{G w \delta \cos ^{2} \theta}{t}+\frac{4}{3} E w \frac{\delta \sin ^{2} \theta}{t}\left(1+\frac{w^{2}}{4 t^{2}}\right),
$$

since $A=w / 2$. It is readily seen that this expression reduces to our result for the infinite strip when $\theta=\pi / 2$.

\subsection{Finite V-shaped blocks}

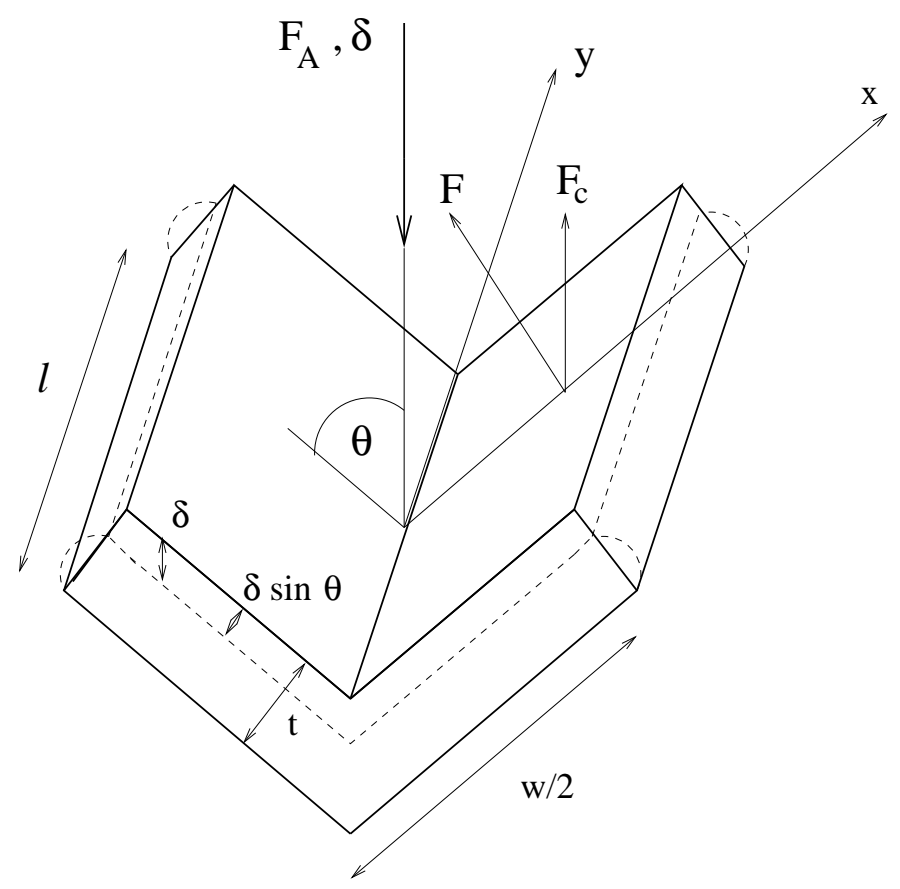

Figure 5: Finite V-shaped block

As we did in the previous example, we consider only one leg of the finite V-shaped block of length $\ell$. As before, the vertical displacement $\delta$ induces compression and shear in the rubber block. We first find the axial component of the compression force. Recall that $F=F_{1}+F_{2}$, where $F_{1}$ comes from the homogeneous compression. We use the empirical factor (2.1) proposed by Gent and Meinecke [3] to obtain

$$
F_{1}=E \delta \sin \theta \frac{w \ell}{2 t}\left(\frac{4}{3}-\frac{2}{3} \frac{w \ell / 4+t^{2}}{w^{2} / 4+\ell^{2} / 4+2 t^{2}}\right) .
$$


$F_{2}$ is again derived by Reynolds' theory. First we have that the pressure $P$ satisfies the following equation:

$$
\begin{aligned}
& \frac{\partial^{2} P}{\partial x^{2}}+\frac{\partial^{2} P}{\partial y^{2}}=-12 \frac{G}{t^{3}} \delta \sin \theta \\
& P=0 \text { at } x=\frac{w}{2}, \quad y= \pm \frac{\ell}{2}, \\
& \frac{d P}{d x}=0 \text { at } x=0 .
\end{aligned}
$$

Let us denote the right side of (4.31) by $M$. Then this equation is analogous to the torsion problem described in [7], and very similar to the compression of a finite rectangular block. Thus we can use arguments similar to those in Section 3 to obtain a solution. The boundary conditions at $x=\frac{w}{2}$ and $x=0$ are satisfied by looking for $P$ in the form of a series

$$
P=\sum_{n=1,3,5, \ldots}^{\infty} \cos \frac{n \pi x}{w} Y_{n}
$$

where $Y_{1}, Y_{3}, \ldots$ are functions of $y$ only. We can argue as in Section 3 to find $Y_{n}$ and $P$.

With routine calculations we thus obtain

$$
P=\frac{12 G}{t^{3}} \delta \sin \theta \sum_{n=1,3,5, \ldots}^{\infty} \frac{4}{n \pi \lambda_{n}^{2}}(-1)^{\frac{n-1}{2}}\left[1-\frac{\cosh \left(\lambda_{n} y\right)}{\cosh \left(\lambda_{n} \ell / 2\right)}\right] \cos \frac{n \pi x}{w},
$$

where $\lambda_{n}^{2}=\frac{n^{2} \pi^{2}}{w^{2}}$. To find $F_{2}$ we integrate $P$ along the bonded plate of length $\ell$

$$
\begin{aligned}
F_{2} & =\int_{-\ell / 2}^{\ell / 2} \int_{0}^{w / 2} P d x d y \\
& =\frac{12 G}{t^{3}} \delta \sin \theta \sum_{n=1,3,5, \ldots}^{\infty}\left[\frac{4 w \ell}{n^{2} \pi^{2} \lambda_{n}^{2}}-\frac{8 w}{n^{2} \pi^{2} \lambda_{n}^{3}} \tanh \frac{\lambda_{n} \ell}{2}\right] .
\end{aligned}
$$

Substituting $\lambda_{n}^{2}=\frac{n^{2} \pi^{2}}{w^{2}}$ and using the fact that $\sum_{n=1,3,5, \ldots}^{\infty} \frac{1}{n^{4}}=\frac{\pi^{4}}{96}$ we obtain

$$
F_{2}=\frac{1}{2 t^{3}} G \delta \sin \theta w^{3} \ell\left(1-\frac{192}{\pi^{5}} \frac{w}{\ell} \sum_{n=1,3,5, \ldots}^{\infty} \frac{1}{n^{5}} \tanh \frac{n \pi \ell}{2 w}\right) .
$$

This series converges rapidly and it can be computed easily for different ratios $\frac{w}{\ell}$. For example if $w=\ell$ (which is not a square cross section of one leg in our notation) we obtain

$$
F_{2}=\frac{0.4217}{2 t^{3}} G \delta \sin \theta w^{4}
$$

For $w / 2=\ell$ (which is a square cross section of one leg in our notation) we find

$$
F_{2}=\frac{0.1716}{2 t^{3}} G \delta \sin \theta \frac{w^{4}}{2}
$$


In the general case the compression force is given by

$$
\begin{aligned}
F & =F_{1}+F_{2}=E \delta \sin \theta \frac{w \ell}{2 t}\left(\frac{4}{3}-\frac{2}{3} \frac{w \ell / 4+t^{2}}{w^{2} / 4+\ell^{2} / 4+2 t^{2}}\right) \\
& +\frac{1}{2 t^{3}} G \delta \sin \theta w^{3} \ell\left(1-\frac{192}{\pi^{5}} \frac{w}{\ell} \sum_{n=1,3,5, \ldots}^{\infty} \frac{1}{n^{5}} \tanh \frac{n \pi \ell}{2 w}\right) \\
& =E \frac{w \ell}{2 t} \delta \sin \theta\left(\frac{4}{3}-\frac{2}{3} \frac{w \ell / 4+t^{2}}{w^{2} / 4+\ell^{2} / 4+2 t^{2}}\right. \\
& \left.+\frac{1}{3 t^{2}} w^{2}\left(1-\frac{192}{\pi^{5}} \frac{w}{\ell} \sum_{n=1,3,5, \ldots}^{\infty} \frac{1}{n^{5}} \tanh \frac{n \pi \ell}{2 w}\right)\right) .
\end{aligned}
$$

The axial component of the compression force is $F_{c}=F \sin \theta$.

The shear force induced by the tangential component of the displacement is given by

$$
F_{t}=\frac{G w \delta \cos \theta}{2 t},
$$

and its axial component is $F_{s}=F_{t} \cos \theta$. Thus we can evaluate $F_{a}=F_{c}+F_{s}$ for different sized blocks. Again the total axial force, $F_{A}=2 F_{a}$, reduces to our result for rectangular blocks when $\theta=\pi / 2$.

\subsection{Extension to slightly compressible V-shaped blocks}

In the above analysis we made the assumption that the rubber is incompressible. In reality rubber is slightly compressible, with a large, but finite bulk modulus. The usual incompressibility assumption stems from the fact that the shear modulus of the rubber is relatively low, about a thousand times smaller than its bulk modulus. Thus rubber accommodates stresses in a way such that the change in volume is negligible compared to its change in shape.

In this case we need to modify our results for the $F_{2}$ component of the compression force. The other forces are not affected by the bulk modulus. The equation we must solve in this case is the following

$$
\begin{aligned}
& \frac{\partial^{2} P}{\partial x^{2}}+\frac{\partial^{2} P}{\partial y^{2}}=-12 \frac{G}{t^{3}} \delta \sin \theta+\frac{12 G}{t^{2} K} P, \\
& P=0 \text { at } x=\frac{w}{2}, y= \pm \frac{\ell}{2}, \\
& \frac{d P}{d x}=0 \text { at } x=0,
\end{aligned}
$$

where $K$ is the bulk modulus of the rubber. Let us again denote the first term on the right side of (4.35) by $M$. We can solve the equation in the same manner as we solved (4.31). The boundary conditions at $x=\frac{w}{2}$ and $x=0$ are satisfied by seeking $P$ in the form of a series:

$$
P=\sum_{n=1,3,5, \ldots}^{\infty} \cos \frac{n \pi x}{w} Y_{n},
$$


where $Y_{1}, Y_{3}, \ldots$ are functions of $y$ only. Arguing as before we obtain that

$$
P=\frac{12 G}{t^{3}} \delta \sin \theta \sum_{n=1,3,5, \ldots}^{\infty} \frac{4}{n \pi \lambda_{n}^{2}}(-1)^{\frac{n-1}{2}}\left[1-\frac{\cosh \lambda_{n} y}{\cosh \lambda_{n} \frac{\ell}{2}}\right] \cos \frac{n \pi x}{w}
$$

with

$$
\lambda_{n}^{2}=\frac{n^{2} \pi^{2}}{w^{2}}+\frac{12 G}{t^{2} K}
$$

To find $F_{2}$ we integrate $P$ along the bonded plate

$$
\begin{aligned}
F_{2} & =\int_{-\ell / 2}^{\ell / 2} \int_{0}^{w / 2} P d x d y \\
& =\frac{12 G}{t^{3}} \delta \sin \theta \sum_{n=1,3,5, \ldots}^{\infty} \frac{4 w \ell}{n^{2} \pi^{2} \lambda_{n}^{2}}-\frac{8 w}{n^{2} \pi^{2} \lambda_{n}^{3}} \tanh \frac{\lambda_{n} \ell}{2} .
\end{aligned}
$$

Once the force $F_{2}$ is obtained the remaining analysis is the same as in the previous subsection since the other forces are not affected by the bulk modulus and the assumption that the rubber is slightly compressible.

\subsection{Finite element analysis results}

Linear finite element analysis was performed using ANSYS 5.6 to verify the theoretical solution for the finite V-shaped block. 3D analysis was performed using Solid95 elements with reduced integration. The material was assumed to be linearly elastic with Young's modulus of $4.137 \cdot 10^{6} \mathrm{~Pa},(600 \mathrm{psi})$ and a Poisson's ratio of 0.4995 (slightly compressible material). To cover a reasonable range of shapes, the angle, $\theta$ was fixed at $\pi / 4$ and $\pi / 3$. The width $w$ was kept at $0.0505 \mathrm{~m},(2$ in), while the thickness varied from 0.0031 to 0.0126 $\mathrm{m},(0.125$ to $0.5 \mathrm{in})$, and the length varied from 0.0126 to $0.203 \mathrm{~m},(0.5$ to 8 inches $)$. A y-displacement of $2.53990 \cdot 10^{-5} \mathrm{~m},(0.001 \mathrm{in})$ was imposed on the top surface to simulate a small compression $\delta$. Figures 6-7 show the comparison of the finite element and the theoretical results for the vertical force needed to compress $\mathrm{V}$-shaped blocks of different angles and sizes by $\delta$ in the slightly compressible case. There is a very good agreement between the two results with a discrepancy of less than $10 \%$. This is quite satisfactory considering that both methods are approximate. The largest discrepancy occured when the length $\ell$ was small and the width $t$ was large. This is not surprising since in that case some of the assumptions of the pressure method do not apply.

\section{Acknowledgments}

We are grateful to Gail G. Licayan for her contributions in organizing some of the material for this paper. This research was supported in part (HTB and GAP) by the Air Force Office of Scientific Research under grants AFOSR F49620-98-1-0180 and AFOSR F49620-01-1-0026. 


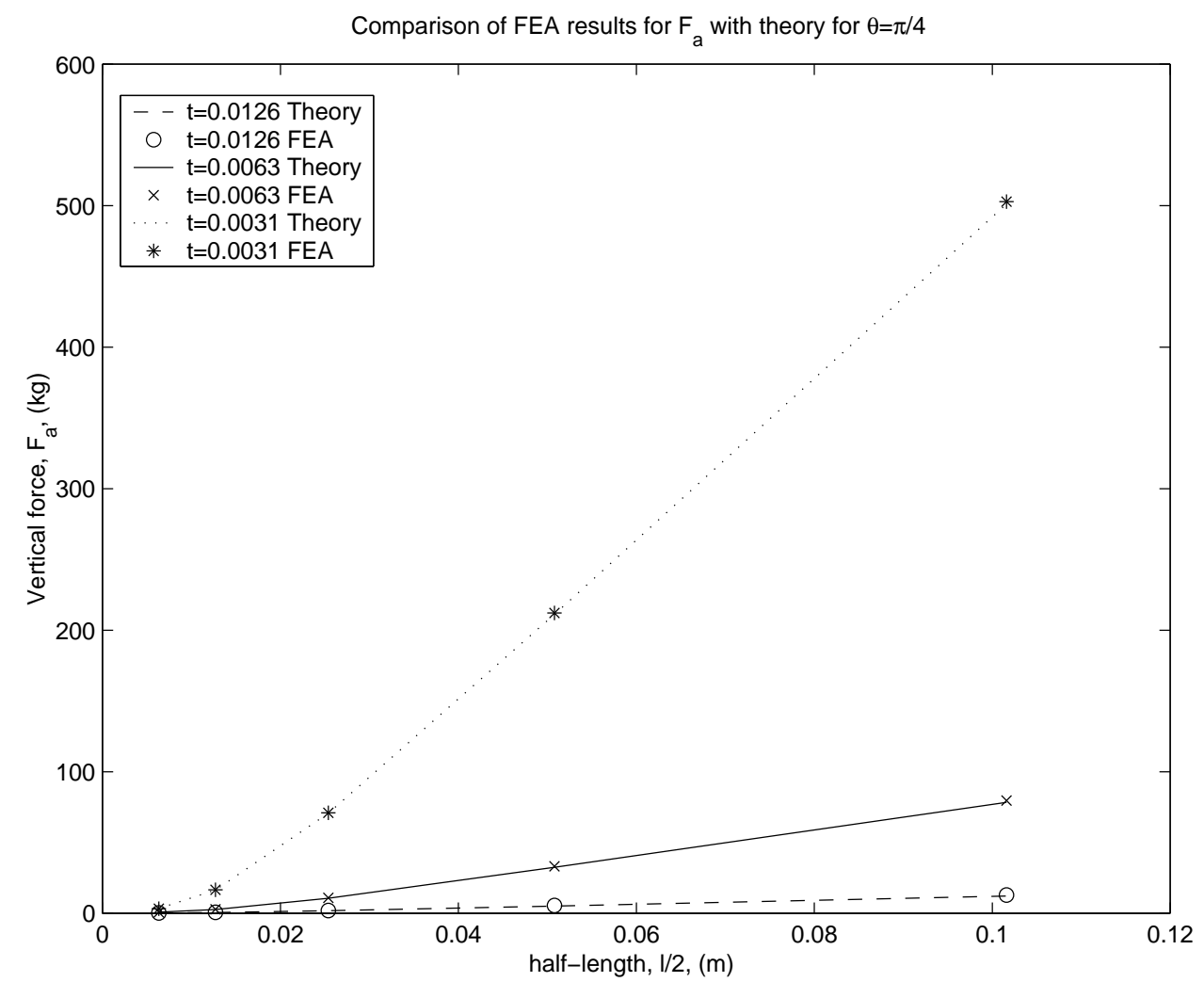

Figure 6: Comparison of FEA results for $F_{a}$ with theory for $\theta=\pi / 4$

\section{References}

[1] H.T. Banks, G.A. Pinter and O.H. Yeoh: Compression of bonded rubber blocks, Rubber Chemistry and Technology, submitted.

[2] A.N. Gent and P.B. Lindley, The compression of bonded rubber blocks, Proc. Instn. Mech. Engrs., 173, (1959) pp. 111-117.

[3] A.N. Gent and E.A. Meinecke, Compression, bending and shear of bonded rubber blocks, Polym. Eng. Sci., 10, (1970) pp. 48-53.

[4] A.N. Gent, Compression of rubber blocks, Rubb. Chem. Technol., 67, (1994) pp.917-931.

[5] M.C. Constantinou, A. Kartoum and J.M. Kelly, Analysis of compression of hollow circular elastomeric bearings, Eng. Struct., 14, (1992), pp. 103-111.

[6] P.B. Lindley, K.G.N. Fuller and A.H. Muhr, Engineering Design with Natural Rubber, Malaysian Rubber Producers' Research Association, Brickendonbury, England, 1992.

[7] S. Timoshenko, J.N. Goodier, Theory of Elasticity 2nd ed. McGraw-Hill, New York, 1951. 


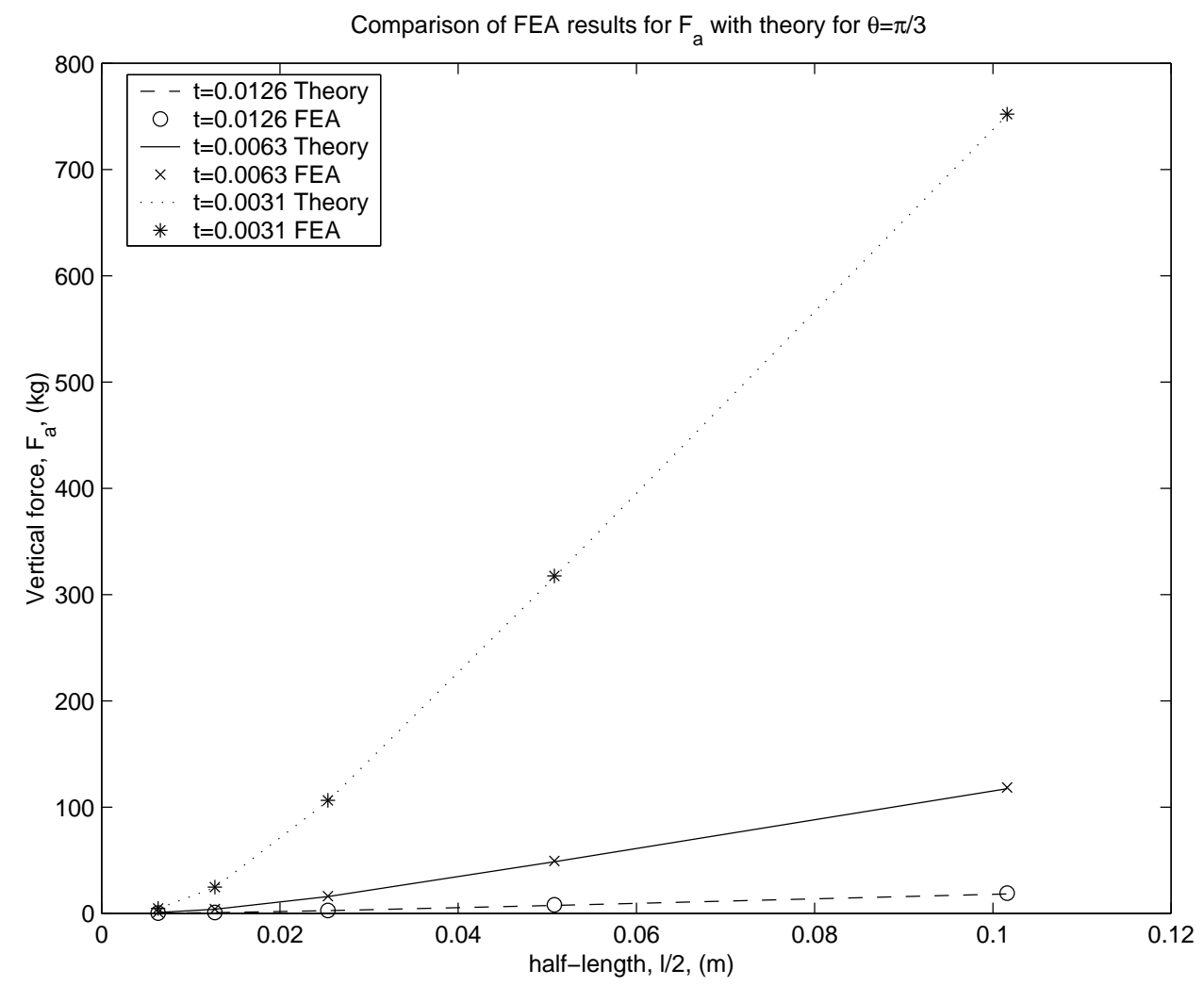

Figure 7: Comparison of FEA results for $F_{a}$ with theory for $\theta=\pi / 3$

[8] T.J. Higgins, A Comprehensive Review of Saint-Venant's Torsion Problem, Am. J. Physics, vol.10.(1942), pp. 248-259.

[9] I.S. Sokolnikoff, On a Solution of Laplace's Equation with an Application to the Torsion Problem for a Polygon with Reentrant Angles, Trans. Am. Math. Soc. vol.33. (1931), pp. 719-732. 